\title{
Pattern Recognition in Interdisciplinary Perception and Intelligence
}

This Special Issue came in our mind to celebrate the 50th anniversary of the field of Artificial Intelligence with the objective to show the interdisciplinary of the fields of Pattern Recognition (PR), Artificial Intelligence (AI) and Perception. Pattern Recognition is a field with a strong relation to Artificial Intelligence however oriented to solve basically engineering problems in a large number of applications, and other systems, for example Perception where is required processing sensory data for its interpretation.

The name of Artificial Intelligence was coined in 1955 when J. McCarthy (Dartmouth College, New Hampshire), M. L. Minsky (Harvard University), N. Rochester (I.B.M. Corporation), and C.E. Shannon (Bell Telephone Laboratories) proposed to hold "Dartmouth summer research project on ARTIFICIAL INTELLIGENCE", known now as the Dartmouth Conference. The Dartmouth Conference was held during the summer of 1956, to discuss various aspects of learning and intelligence that could be simulated on machines. PR had been one of the important fields in AI [Minsky 1961] till the beginning of 70's when the first IJCPR (International Joint Conference on Pattern Recognition) was organised. The name of Pattern Recognition appeared later on with the objective of developing techniques in the area of classification, oriented to solve engineering problems. Both areas share objectives and applications that can be solved in different ways.

Pattern Recognition is the research area studying the operation and design of systems that recognise patterns in data. It encloses sub disciplines like discriminant analysis, feature extraction, error estimation, cluster analysis (together sometimes called statistical pattern recognition); grammatical inference, parsing and matching (sometimes called syntactical and structural pattern recognition). Pattern Recognition is largely related to others techniques as Computer Vision, Fuzzy Sets, Neural Networks and Kernels Classifiers, and to fields like Speech Recognition and Biological Perception, among others.

Pattern Recognition is a discipline that started at the beginning of 1960's [Sebestyen 1962, Nilsson 1965, Fu 1968, Fukunaga 1972, Meisel 1972, Duda-Hart 1973] in the area of statistical pattern recognition, focusing on the development of techniques based on the statistical and probability theory. Several incipient problems were first studied using these techniques, for example, learning theory was first analyzed as statistical pattern recognition problem [Nilsson 1965] and the first models to explain biological neural nets, Perceptrons [Rosenblatt, 1962, Minsky-Papert 1969], were also based on those techniques. The field of Pattern Recognition was then extended to new techniques that allow to describe patterns by a set of repetitive primitives, based on grammar theory [Fu 1974] and matching structured patterns (strings, trees and graphs) [Pavlidis 19721977, Bunke Sanfeliu 1990]. In the last years, new statistical techniques has became popular in the field, Bayesian Networks and Kernel classifiers, where Supported Vector Machines (SVM) is one of the most well know techniques of the last one.

The field of Pattern Recognition very soon became related to Perception because of the similarity of the underlying processes in both disciplines. Perception is defined in psychology and the cognitive sciences, as the process of acquiring, interpreting, 
selecting, and organizing sensory information. Pattern Recognition is defined as the process of acquiring, processing, extracting features and classifying the patterns of input patterns, for example from sensors. In both disciplines the sensory information is acquired, processed and the interpreted (classified) to give an output that in the case of perception systems will be used for interpreting, selecting or organizing.

Perception is a basic system in human beings to learn and interact with the world, where all the sensory systems are involved (vision, auditory, tactile, smell and taste sensors). Although other Perception systems may be useful, they are not involved in human beings (for example, infrared or ultraviolet vision). Vision was one of the earliest Perception systems to be studied, due to its large scope of applicability. The first work on vision was in the decade of the 50's and the first system to recognize 3D objects was done by L. Roberts [Roberts, 1963]. Later, 3D scene analysis (blocks or indoor and outdoor scenes) became popular in AI. Optical character reading was also another of the pioneer works on vision due to the important application on reading the addresses in envelops for automatic classification [Fischer et all 1962].

At present, Pattern Recognition is in almost all the areas of Perception, where vision systems are probably the most known, and they are used in biometrics, industry, medicine, space, robotics, natural science, etc. Although that, speech recognition systems have also became very popular for interactive helping systems in telephonic systems. During the last years, multimodal human interaction has increased attention due to the necessity of tools for human-robot interaction in dynamic and changing environments. In this area, Pattern Recognition and Computer Vision are basic techniques, and examples of them are interactive translation and transcription of music and text images, interactive retrieval of multimedia content, interactive face recognition and other biometric applications, medical imaging assisted diagnosis, computer assisted document layout analysis, multimodal dialog systems (for mobile telephones e information access devices like PDAs), cooperative tracking and recognition of human actions or assisted vehicle driving and human robot interaction.

This Special Issue not only presents some novel works on Pattern Recognition, Artificial Intelligence, and Perception but also on application of PR techniques (such as statistical PR theory) or clustering to problems in AI (such as machine translation, news classification, or social pattern classification).

Antonio Fernández-Caballero

Alberto Sanfeliu

Yoshiaki Shirai

Guest Editors

\section{References}

[Minsky, 1961] M. Minsky, "Steps Toward Artificial Intelligence", Proc. IRE, Vol. 49, No. 1, pp. 8-30, 1961.

[Sebestyen, 1962] G. S. Sebestyen, "Decision Processes in Pattern Recognition". New York: Macmillan, 1962. 
[Fischer et all., 1962] G. L. Fischer, Jr., D. K. Pollock, B. Radack, and M. E. Stevens, Eds., "Optical Character Recognition". Washington, DC: Spartan, 1962.

[Rosenblatt, 1962] F. Rosenblatt, "Principles of Neurodynamics",

New York: Spartan, 1962.

[Roberts, 1963] L. G. Roberts, "Machine Perception of Three-Dimensional Solids", Optical and Electro-Optical Information Processing, pp.159-197, MIT Press, MA: 1963. [Nilsson, 1965] N. J. Nilsson, "Learning Machines-Foundations of Trainable

Pattern-Classifying Systems". New York: McGraw-Hill, 1965.

[Fu, 1968] K. S. Fu, "Sequential Methods in Pattern Recognition and Machine Learning". New York: Academic, 1968.

[Minsky-Papert, 1969] M. Minsky and S. Papert, "Perceptrons". Cambridge, MA: M.I.T. Press, 1969.

[Rosenfeld, 1969] A. Rosenfeld, "Picture Processing by Computer". New York: Academic, 1969.

[Pavlidis, 1972] T. Pavlidis, "Structural pattern recognition: primitives and juxtaposition relations". In: Watanabe, M.S. (Ed.), Frontiers of Pattern Recognition. 1972.

[Fukunaga, 1972] K. Fukunaga, "Introduction to Statistical Pattern Recognition".

New York: Academic, 1972.

[Meisel, 1972] W. Meisel, "Computer-Oriented Approaches to Pattern Recognition".

New York: Academic, 1972.

[Duda-Hart, 1973] R. 0. Duda and P. E. Hart, "Pattern Classification and Scene

Analysis". New York: Wiley, 1973.

[Fu, 1974] K.S. Fu, "Syntactic Methods in Pattern Recognition", Academic Press, New York, 1974.

[Pavlidis, 1977] T. Pavlidis, "Structural Pattern Recognition", Springer-Verlag, New York, 1977.

[Bunke-Sanfeliu, 1990] H. Bunke and A. Sanfeliu, "Syntactic and Structural Pattern Recognition - Theory and Applications". World Scientific Publishing Co. Pte. Ltd.. Series in Computer Science - Vol. 7. 1990. 